\title{
INTEGRATING VOLATILITY CLUSTERING INTO EXPONENTIAL LÉVY MODELS
}

\author{
CHRISTIAN BENDER, ${ }^{*}$ Technische Universität Braunschweig \\ TINA MARQUARDT, ${ }^{* *}$ Munich University of Technology
}

\begin{abstract}
We introduce a class of stock models that interpolates between exponential Lévy models based on Brownian subordination and certain stochastic volatility models with Lévydriven volatility, such as the Barndorff-Nielsen-Shephard model. The driving process in our model is a Brownian motion subordinated to a business time which is obtained by convolution of a Lévy subordinator with a deterministic kernel. We motivate several choices of the kernel that lead to volatility clusters while maintaining the sudden extreme movements of the stock. Moreover, we discuss some statistical and path properties of the models, prove absence of arbitrage and incompleteness, and explain how to price vanilla options by simulation and fast Fourier transform methods.
\end{abstract}

Keywords: Convoluted Lévy process; financial modeling; subordination; volatility clustering

2000 Mathematics Subject Classification: Primary 91B28

Secondary 60G51; 60H05; 26A33

\section{Introduction}

Since the mid 1990s, the modeling of stocks by exponential Lévy processes has gained ever increasing importance. Among the most popular models of pure jump type are the variance gamma process [14], models based on generalized hyperbolic processes [10], and those based on normal tempered stable processes [8]. In particular, Carr et al. [8] studied the subclass of normal tempered stable processes, which can be obtained by subordinating a Brownian motion with drift to an increasing Lévy process (subordinator) of tempered stable type. This popular class of processes is often referred to as CGMY models.

A main advantage of exponential Lévy processes is that they are able to capture extreme movements of stock prices by choosing a distribution with fat tails. However, they still exhibit, by definition, independent increments. Therefore, exponential Lévy models cannot produce volatility clusters. This regularly observed feature of financial time series means that large movements of the stock prices tend to be followed by other large movements (while small movements tend to be followed by small movements). Therefore, it was suggested in [8] to run an exponential Lévy process in a business time, which is an increasing process independent of the original Lévy process. Typical choices for the business time are an integrated square root diffusion (Cox-Ingersoll-Ross process) or an integrated Ornstein-Uhlenbeck process driven by a Lévy subordinator, as studied by Barndorff-Nielsen and Shephard [1]. While introducing

Received 11 April 2008; revision received 29 June 2009.

* Current address: Department of Mathematics, Saarland University, PO Box 151150, D-66041 Saarbrücken, Germany.

Email address: bender@math.uni-sb.de

** Postal address: Center of Mathematical Sciences, Munich University of Technology, D-85747 Garching, Germany.

Email address: marquard@ma.tum.de 
the business time process can cause volatility clusters, it comes at the cost of requiring an additional source of randomness which complicates the model.

In the present paper we suggest a different way to incorporate volatility clusters into exponential Lévy models based on Brownian subordination, without adding an additional source of randomness. To this end, let $W$ denote a Brownian motion and let $L$ denote an increasing Lévy process. Given a deterministic kernel function $k(t, s)$, we integrate memory into the subordinator $L$ by a convolution integral $T(t)=\int_{0}^{t} k(t, s) \mathrm{d} L(s)$. This type of process is related to fractional Lévy processes and convoluted Lévy processes, as studied in [16] and [3], respectively. We then run the Brownian motion in the business time $T$, i.e. we consider $X(t)=W(T(t))$ as the driving process of the model. In Section 2 we motivate and illustrate some explicit choices for the kernel function, partly based on fractional integral operators, that induce different kinds of memory effect. In contrast to the original exponential Lévy model, these kernels yield models with continuous trajectories, but the Lévy model comes up as a limiting case.

In general, our assumptions guarantee that the business time $T$ is a strictly increasing, continuous process, i.e. the jumps of the Lévy process $L$ are smoothed out to some extent by the convolution. Depending on the choice of the kernel, we can build models which have rougher paths than a Brownian motion with stochastic volatility (which typically has a Hölder continuity index of $\frac{1}{2}$ ), but do not exhibit jumps. We note that such path behavior is supported by some empirical studies making use of the singularity spectrum, as reported in, e.g. [9, Chapter 7.6].

The paper is organized as follows. In Section 2 we introduce the concept of a convoluted subordinator as a business time. Some basic properties (including the computation of the second-order structure and of the characteristic function) are deduced and several examples of convolution kernels are provided. We also show that many stochastic volatility models with Lévy-driven volatility such as the Barndorff-Nielsen-Shephard model [1] can be cast into the framework of convoluted subordinators. In Section 3 we discuss some statistical properties of the associated stock model, which is basically the stochastic exponential of the Brownian motion $W$ in the business time $T$. Section 4 is devoted to the proof of absence of arbitrage and incompleteness. Finally, in Section 5 we explain the pricing of vanilla options in this model class using simulation or fast Fourier transform methods. To this end, we compute the log-characteristic function of the stock model and simplify it analytically in some specific examples. We illustrate the implied volatility smile of the models and compare it with one of the original exponential Lévy models.

\section{Convoluted subordinators as the business time}

In this section we discuss the class of business times which we consider in this paper. We start with some basic facts on Lévy subordinators. A Lévy subordinator is an increasing Lévy process $\{L(t)\}_{t \geq 0}$ on a filtered probability space $\left(\Omega, \mathcal{F},\left(\mathcal{F}_{t}\right)_{t \geq 0}, \mathrm{P}\right)$ satisfying the usual conditions. In terms of the characteristic triplet $\left(\gamma, \sigma^{2}, \nu\right)$, where

$$
\begin{gathered}
\mathrm{E}\left[\mathrm{e}^{\mathrm{i} u L(t)}\right]=\mathrm{e}^{t \psi(u)}, \\
\psi(u)=\mathrm{i} \gamma u-\frac{1}{2} u^{2} \sigma^{2}+\int_{\mathbb{R} \backslash\{0\}}\left(\mathrm{e}^{\mathrm{i} u x}-1-\mathrm{i} u x \mathbf{1}_{\{|x| \leq 1\}}\right) \nu(\mathrm{d} x), \quad u \in \mathbb{R},
\end{gathered}
$$


a subordinator can be characterized by

$$
\sigma=0, \quad v((-\infty, 0))=0, \quad \int_{0}^{\infty}(x \wedge 1) v(\mathrm{~d} x)<\infty, \quad \gamma_{0}:=\gamma-\int_{0}^{1} x v(\mathrm{~d} x) \geq 0 .
$$

Hence, in terms of $\gamma_{0}$, the characteristic exponent $\psi$ of a subordinator can be simplified to

$$
\psi(u)=\mathrm{i} \gamma_{0} u+\int_{0}^{\infty}\left(\mathrm{e}^{\mathrm{i} u x}-1\right) v(\mathrm{~d} x), \quad u \in \mathbb{R} .
$$

Moreover, $L$ has a finite second moment if and only if $\int_{x>1} x^{2} v(\mathrm{~d} x)<\infty$. In this case it holds that

$$
\operatorname{var}(L(1))=\int_{x>0} x^{2} v(\mathrm{~d} x) .
$$

For the proofs and more information about Lévy subordinators, we refer the reader to [9] or [20]. We also note that integration with respect to $L$ can be performed pathwise, because $L$ is increasing.

Subordinators widely used in financial modeling include the gamma subordinator, given by $(\kappa>0)$

$$
\gamma_{0}=0, \quad v_{\kappa}(A)=\int_{A} \frac{\mathrm{e}^{-x / \kappa}}{\kappa x} \mathrm{~d} x,
$$

and tempered stable subordinators with index of stability $0<\alpha<1$, given by

$$
\gamma_{0}=0, \quad v_{\kappa, \alpha}(A)=\int_{A} \frac{1}{\Gamma(1-\alpha)}\left(\frac{1-\alpha}{\kappa}\right)^{1-\alpha} \frac{\mathrm{e}^{-(1-\alpha) x / \kappa}}{x^{1+\alpha}} \mathrm{d} x .
$$

In both cases the subordinators are already normalized in the sense that

$$
\mathrm{E}[L(1)]=\gamma_{0}+\int_{x>0} x v(\mathrm{~d} x)=1 .
$$

We note that both examples are subordinators of infinite activity, that is, $v((0, \infty))=\infty$.

Throughout the paper, the following hypothesis will be in force.

Assumption 1. L is a Lévy subordinator for which $\gamma_{0}>0$ or $v((0, \infty))=\infty$ holds, i.e. $L$ is strictly increasing.

A convoluted subordinator can now be defined by convolution of a subordinator with a Volterra-type kernel.

Definition 1. A stochastic process $\{T(t)\}_{t \geq 0}$ defined by

$$
T(t)=\int_{0}^{t} k(t, s) \mathrm{d} L(s)
$$

is called a convoluted subordinator if $L$ is a Lévy subordinator satisfying Assumption 1 and $k:[0, \infty)^{2} \rightarrow[0, \infty)$ fulfills the following properties.

(i) For fixed $t \in[0, \infty)$, the mapping $s \mapsto k(t, s)$ is integrable.

(ii) For fixed $s \in[0, \infty)$, the mapping $t \mapsto k(t, s)$ is continuous and increasing. Moreover, there is an $\varepsilon>0$ such that $t \mapsto k(t, s)$ is strictly increasing on $[s, s+\varepsilon]$.

(iii) $k(t, s)=0$, whenever $s>t \geq 0$, i.e. the kernel $k$ is of Volterra type. 
The next proposition collects some path properties of a convoluted subordinator.

Proposition 1. The process $T=\{T(t)\}_{t \geq 0}$ has almost surely continuous and strictly increasing sample paths.

Proof. Recalling that $L$ is strictly increasing by Assumption 1, it follows directly from Definition 1(ii) that $T$ has strictly increasing sample paths. To show continuity from below, we consider, for $t>u \geq 0$,

$$
\begin{aligned}
T(t)-T(u) & =\int_{0}^{t} k(t, s) \mathrm{d} L(s)-\int_{0}^{u} k(u, s) \mathrm{d} L(s) \\
& =\int_{0}^{t}(k(t, s)-k(u, s)) \mathrm{d} L(s)-\int_{u}^{t} k(u, s) \mathrm{d} L(s) .
\end{aligned}
$$

Since $k(t, s)-k(u, s)$ is strictly decreasing in $u$, the first integral tends to 0 as $u \nearrow t$ by monotone convergence. By the dominated convergence theorem, the second term converges to 0 as $u \nearrow t$, since $k(u, s) \leq k(t, s)$ for $u<t$. Using a similar argument, we can show continuity from above and the proof is complete.

Since $T$ is increasing, it qualifies as a random time change and can be applied as a business time. Note also that the convolution with the Volterra-type kernel $k$ smoothes the jumps of $L$ and, thus, results in a continuous time change.

Before we present some examples, we briefly discuss some distributional properties of the business time $T$ in terms of the kernel $k$. The proof of the following theorem is given in [21].

Theorem 1. For every $t \in[0, \infty)$, the distribution of $T(t)$ is infinitely divisible and

$$
\mathrm{E}[\exp \{z T(t)\}]=\exp \left\{\phi_{t}(z)\right\}
$$

where

$$
\phi_{t}(z)=z \gamma_{0} \int_{0}^{t} k(t, s) \mathrm{d} s+\int_{0}^{t} \int_{0}^{\infty}\left(\mathrm{e}^{z k(t, s) x}-1\right) v(\mathrm{~d} x) \mathrm{d} s,
$$

holds for $z=u+\mathrm{i} v$ with $v \in \mathbb{R}$ and $u \leq 0$.

From the previous theorem we can easily extract information about the moments of the business time $T$.

Corollary 1. (Moments.) (i) If $\mathrm{E}[L(1)]<\infty$, we have

$$
\mathrm{E}[T(t)]=\mathrm{E}[L(1)] \int_{0}^{t} k(t, s) \mathrm{d} s .
$$

(ii) If $\mathrm{E}\left[L(1)^{2}\right]<\infty$, it holds that

$$
\operatorname{cov}(T(t), T(u))=\mathrm{E}\left[L(1)^{2}\right] \int_{0}^{t \wedge u} k(t, s) k(u, s) \mathrm{d} s .
$$

Furthermore, for $0 \leq t_{1} \leq t_{2} \leq t_{3} \leq t_{4}$,

$$
\begin{aligned}
\operatorname{cov}\left(T\left(t_{4}\right)-T\left(t_{3}\right), T\left(t_{2}\right)-T\left(t_{1}\right)\right)= & \mathrm{E}\left[L(1)^{2}\right] \int_{0}^{t_{2}} k\left(t_{2}, s\right)\left(k\left(t_{4}, s\right)-k\left(t_{3}, s\right)\right) \mathrm{d} s \\
& +\mathrm{E}\left[L(1)^{2}\right] \int_{0}^{t_{1}} k\left(t_{1}, s\right)\left(k\left(t_{3}, s\right)-k\left(t_{4}, s\right)\right) \mathrm{d} s .
\end{aligned}
$$


As a first example, we consider a fractional subordinator.

Example 1. (Fractional subordinator.) For $H>\frac{1}{2}$, we consider the kernel

$$
z_{H}(t, s)=d_{H} s^{1 / 2-H} \int_{s}^{t}(u-s)^{H-3 / 2} u^{H-1 / 2} \mathrm{~d} u,
$$

with a normalizing constant

$$
d_{H}=\left(H-\frac{1}{2}\right)\left(\frac{2 H \Gamma(3 / 2-H)}{\Gamma(H+1 / 2) \Gamma(2-2 H)}\right)^{1 / 2} .
$$

We call the process $T^{(H)}(t):=\int_{0}^{t} z_{H}(t, s) \mathrm{d} L(s)$ a fractional subordinator with Hurst parameter $H$ driven by $L$. If $L$ has a finite second moment and is normalized to $\mathrm{E}\left[L^{2}(1)\right]=1$, we can deduce, from the previous corollary and the representation formula for a fractional Brownian motion on an interval (see [2] or [18]), that $T^{(H)}$ has the same second-order structure as a fractional Brownian motion with Hurst parameter $H$. In particular, the autocovariance function

$$
\begin{aligned}
\rho_{H}(n) & =\operatorname{cov}\left(T^{(H)}(k)-T^{(H)}(k-1), T^{(H)}(k+n)-T^{(H)}(k+n-1)\right) \\
& =\frac{1}{2}\left(|n+1|^{2 H}-2|n|^{2 H}+|n-1|^{2 H}\right) \\
& \sim H(2 H-1)|n|^{2 H-1}
\end{aligned}
$$

exhibits long memory. Roughly speaking, this can be interpreted as follows. A large jump of $L$ acts as a shock to the market which increases the trading activity. However, the shock does not vanish instantaneously, but the aftereffects of the shock persist and influence the market later on.

Note that, from the previous corollary and by substitution to a beta integral, we can also calculate

$$
\mathrm{E}[T(t)]=\mathrm{E}[L(1)] d_{H} \int_{0}^{t} s^{1 / 2-H} \int_{s}^{t}(u-s)^{H-3 / 2} u^{H-1 / 2} \mathrm{~d} u \mathrm{~d} s=C_{H} t^{2 H}
$$

for some constant $C_{H}$. This implies that the mean cumulated trading activity increases faster than linearly. This property is questionable, at least when $H \gg 0.5$. The next example will mend this problem.

Example 2. (Holmgren-Liouville fractional subordinator.) A damped version of the fractional Holmgren-Liouville integral of the indicator function leads to the kernel

$$
\tilde{z}_{H}(t, s)=\frac{H+1 / 2}{\mathrm{E}[L(1)]}\left(1-\frac{s}{t}\right)_{+}^{H-1 / 2}
$$

provided $L$ satisfies $\mathrm{E}[L(1)]<\infty$. Here $(\cdot)_{+}$denotes the positive part. The corresponding business time driven by $L$ is $\tilde{T}^{(H)}(t)=\int_{0}^{t} \tilde{z}_{H}(t, s) \mathrm{d} L(s)$.

This kernel is much simpler than the one in the previous example, but has a similar qualitative behavior. If a large jump of $L$ occurs at time $s$ then the business time will increase with a gradient equal to $\infty$ at $t=s$. This corresponds to a sudden shock to the market. Then gradually the gradient decreases, but weak aftereffects of the jump influence the increments of the business 


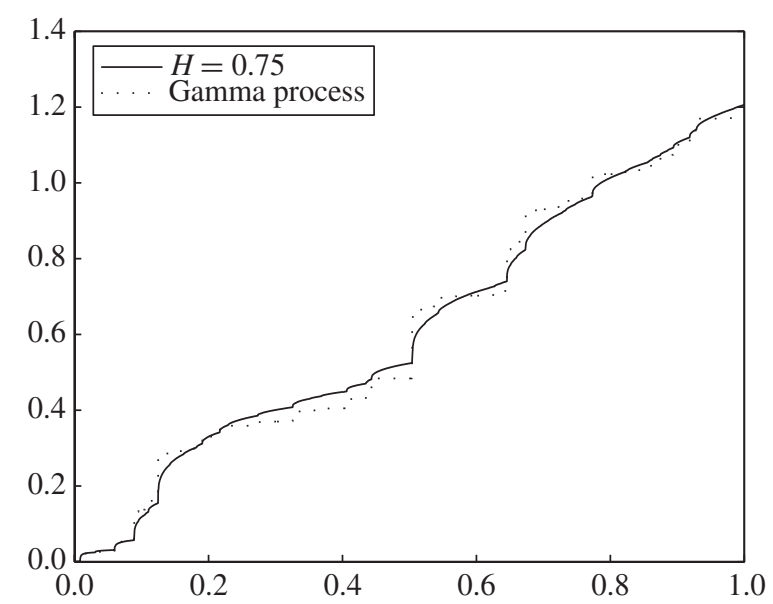

FIGURE 1: Comparison between a sample path of a gamma process $L$ with $\kappa=\frac{1}{20}$ and its convolution $\tilde{T}^{(H)}$ with a damped Holmgren-Liouville kernel with $H=\frac{3}{4}$.

time forever. Contrary to the fractional subordinator, there is no simple expression known for the second-order structure, but we can easily calculate

$$
\begin{gathered}
\mathrm{E}\left[\tilde{T}^{(H)}(t)\right]=\left(H+\frac{1}{2}\right) \int_{0}^{t}\left(1-\frac{s}{t}\right)_{+}^{H-1 / 2} \mathrm{~d} s=t, \\
\operatorname{var}\left(\tilde{T}^{(H)}(t)\right)=\frac{(H+1 / 2)^{2} \mathrm{E}\left[L(1)^{2}\right]}{\mathrm{E}[L(1)]^{2}} \int_{0}^{t}\left(1-\frac{s}{t}\right)_{+}^{2 H-1} \mathrm{~d} s=\frac{(H+1 / 2)^{2}}{2 H} \frac{\mathrm{E}\left[L(1)^{2}\right]}{\mathrm{E}[L(1)]^{2}} t .
\end{gathered}
$$

Figure 1 compares a sample path of $\tilde{T}^{H}$ and the corresponding path of the driving gamma process.

Example 3. As a third example, we present a simple kernel which exhibits short memory effects, namely, for $\varepsilon>0$,

$$
k_{\varepsilon}(t, s)=\mathrm{E}[L(1)]^{-1} \min \left(1, \frac{(t-s)_{+}}{\varepsilon}\right),
$$

and the business time $T_{\varepsilon}(t)=\int_{0}^{t} k_{\varepsilon}(t, s) \mathrm{d} L(s)$ associated to a Lévy subordinator $L$. Here, a jump of $L$ at time $s$ increases the trading activity in the market linearly (with the jump height divided by $\varepsilon$ as gradient) for a short period of length $\varepsilon$. We will see in Section 5 that the simple form of the kernel facilitates efficient option pricing via fast Fourier transform. Note also that integration by parts yields

$$
T_{\varepsilon}(t)=\frac{1}{\mathrm{E}[L(1)] \varepsilon} \int_{\max \{0, t-\varepsilon\}}^{t} L(s) \mathrm{d} s .
$$

Concerning the moments of $T_{\varepsilon}$, we note that

$$
\mathrm{E}\left[T_{\varepsilon}(t)\right]= \begin{cases}t-\frac{\varepsilon}{2}, & t \geq \varepsilon, \\ \frac{t^{2}}{2 \varepsilon}, & t<\varepsilon,\end{cases}
$$




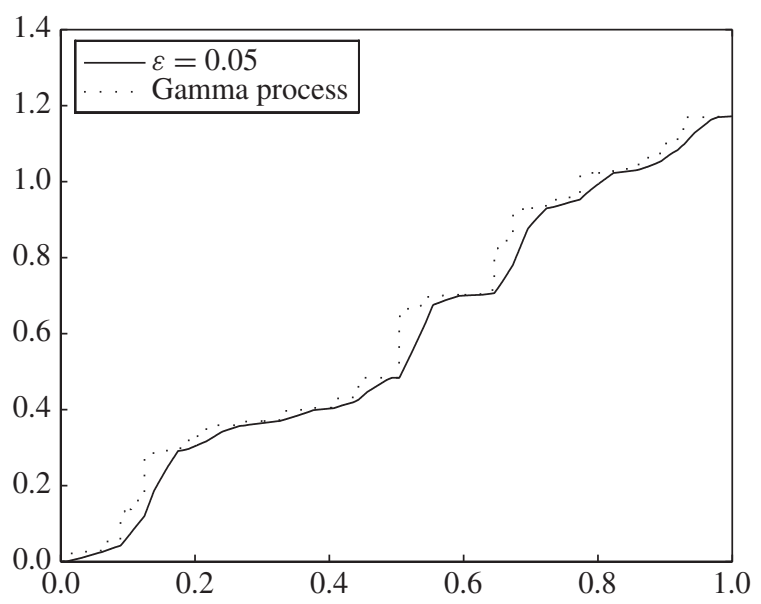

FIGURE 2: Comparison between a sample path of a gamma process $L$ with $\kappa=\frac{1}{20}$ and its convolution $T_{\varepsilon}$ with the kernel $k_{\varepsilon}$ for $\varepsilon=\frac{1}{20}$.

$$
\operatorname{var}\left(T_{\varepsilon}(t)\right)=\frac{\mathrm{E}\left[L(1)^{2}\right]}{\mathrm{E}[L(1)]^{2}} \begin{cases}t-\frac{2 \varepsilon}{3}, & t \geq \varepsilon, \\ \frac{t^{3}}{3 \varepsilon^{2}}, & t<\varepsilon,\end{cases}
$$

and that

$$
\operatorname{cov}\left(T_{\varepsilon}\left(t_{2}\right)-T_{\varepsilon}\left(t_{1}\right), T_{\varepsilon}\left(t_{4}\right)-T_{\varepsilon}\left(t_{3}\right)\right)=0, \quad t_{4} \geq t_{3} \geq t_{2}+\varepsilon \geq t_{2} \geq t_{1} .
$$

The last property explains why we call this kernel a short memory kernel. The correlation of the corresponding business time dies out, if the lag between the increments is larger than $\varepsilon$. This property holds true for any kernel $k(t, s)$ satisfying $k(t, s)=c$ for some constant $c$ and every $(t, s)$ such that $t-s \geq \varepsilon$, thanks to Corollary 1(ii). A typical sample path of $T_{\varepsilon}$ and the corresponding path of the driving gamma process are illustrated in Figure 2.

We close this section with an example of how popular stochastic volatility models driven by Lévy processes can be cast into the framework of convoluted subordinators.

Example 4. (CARMA-type processes.) Suppose that $W$ is a Brownian motion and that $\sigma(t)$ is an adapted process independent of $W$. We interpret $\sigma$ as the stochastic volatility and consider the process $Y(t)=\int_{0}^{t} \sigma(u) \mathrm{d} W(u)$ as the driving process for a stock model. By the DambisDubins-Schwarz theorem (see [12, Theorem 3.4.6]), $Y$ has the same law as the time-changed Brownian motion $\tilde{Y}(t)=W(T(t))$, where

$$
T(t)=\langle Y\rangle_{t}=\int_{0}^{t} \sigma^{2}(u) \mathrm{d} u .
$$

Suppose now that

$$
\sigma^{2}(t)=\sigma^{2}(0) h(t)+\int_{0}^{t} h(t-s) \mathrm{d} L(s),
$$

where $L$ is a Lévy subordinator, $\sigma^{2}(0)$ is independent of $L$, and $h$ is a deterministic function satisfying $h(u)=0$ for $u \leq 0$ and $h(u)>0$ for $u>0$. This setting subsumes the 
Barndorff-Nielsen-Shephard model [1] and CARMA-type processes as volatility; see [5] and [6]. Different choices for the function $h$ are also discussed in [11]. If $h$ is bounded, we can interchange the order of integration and obtain

$$
T(t)-\sigma^{2}(0) \int_{0}^{t} h(u) \mathrm{d} u=\int_{0}^{t} \int_{0}^{u} h(u-s) \mathrm{d} L(s) \mathrm{d} u=\int_{0}^{t}\left(\int_{s}^{t} h(u-s) \mathrm{d} u\right) \mathrm{d} L(s) .
$$

Therefore, $T$ is basically a convoluted subordinator with kernel $k(t, s)=\int_{s}^{t} h(u-s) \mathrm{d} u$. None of the kernels from the previous examples are of this special form. Nonetheless, there is an important difference between the short memory kernel of Example 3 and the fractional kernels. The business time induced by the short memory kernel $k_{\varepsilon}$ can be obtained from a stochastic volatility model with

$$
\sigma^{2}(t)=\frac{L(t)-L((t-\varepsilon) \vee 0)}{\varepsilon} .
$$

Contrarily, the fractional kernels yield business times which are continuous, but not Lipschitz continuous and can therefore not be recovered by stochastic volatility models (with nonexploding $\sigma$ ).

\section{Financial models based on convoluted subordinators}

We now introduce a financial market model based on convoluted subordination. It consists of one riskless asset $B$ (bond) and one risky asset $S$ (stock) that operate in uncertain conditions of probabilistic character described by a filtered probability space $\left(\Omega, \mathcal{F},\left(\mathcal{F}_{t}\right)_{t \geq 0}, \mathrm{P}\right)$. Here, the filtration $\left(\mathcal{F}_{t}\right)_{t \geq 0}$ describes the flow of incoming information. Our main assumption about the price process $S$ of the stock is the following. We assume that $S$ satisfies

$$
S(t)=S(0) \exp \left\{r t+\left(\mu-\frac{\sigma^{2}}{2}\right) T(t)+\sigma W(T(t))\right\}, \quad t \geq 0,
$$

where $W=\{W(t)\}_{t \geq 0}$ is a standard Brownian motion and the convoluted subordinator $T=\{T(t)\}_{t \geq 0}$ serves as a business time. Moreover, $\sigma>0, \mu \in \mathbb{R}$, and $S(0)>0$ are constants. The constant $\mu$ models the excess return of the stock (in dependence of the business time) compared to the riskless interest rate $r \geq 0$. Consequently, the riskless bond is given by

$$
B(t)=\mathrm{e}^{r t}, \quad t \geq 0
$$

The basic cumulated noise source of the model is

$$
X(t)=W(T(t)), \quad t \geq 0 .
$$

Some important albeit simple properties of $X$ are stated in the following proposition.

Proposition 2. X satisfies the following properties.

(i) For all $p>0$ and $t>s \geq 0$, we have

$$
\mathrm{E}\left[|X(t)-X(s)|^{p}\right]=\frac{2^{p / 2}}{\pi^{1 / 2}} \Gamma\left(\frac{p+1}{2}\right) \mathrm{E}\left[|T(t)-T(s)|^{p / 2}\right] .
$$

Moreover, for all $n \in \mathbb{N}$,

$$
\mathrm{E}\left[(X(t)-X(s))^{2 n-1}\right]=0
$$


In particular,

$$
\mathrm{E}[X(t)]=0, \quad \operatorname{var}(X(t))=\mathrm{E}[T(t)]=\mathrm{E}[L(1)] \int_{0}^{t} k(t, s) \mathrm{d} s .
$$

(ii) $X$ is leptokurtic if $T$ is nondeterministic.

(iii) X has uncorrelated increments.

(iv) $X$ has weakly stationary increments on an interval $[a, b]$ if and only if $t \mapsto \int_{0}^{t} k(t, s) \mathrm{d} s$ is a linear function on this interval.

(v) X has continuous trajectories.

(vi) The realized volatility satisfies $[X]_{t}=\langle X\rangle_{t}=T(t)$.

Proof. Part (i) can be easily calculated by conditioning on the $\sigma$-field $\mathcal{F}_{\infty}^{T}$ generated by $\{T(t)\}_{t \geq 0}$. For example,

$$
\begin{aligned}
\mathrm{E}\left[|X(t)-X(s)|^{p}\right] & =\mathrm{E}\left[\mathrm{E}\left[|W(T(t))-W(T(s))|^{p} \mid \mathcal{F}_{\infty}^{T}\right]\right] \\
& =\frac{2^{p / 2}}{\pi^{1 / 2}} \Gamma\left(\frac{p+1}{2}\right) \mathrm{E}\left[|T(t)-T(s)|^{p / 2}\right],
\end{aligned}
$$

because $\left(W(T(t))-W(T(s)) \mid \mathcal{F}_{\infty}^{T}\right)$ is normally distributed with zero mean and variance $T(t)-T(s)$.

(ii) From (i) we can calculate the kurtosis

$$
\kappa(X)=\frac{\mathrm{E}\left[X(t)^{4}\right]}{\mathrm{E}\left[X(t)^{2}\right]^{2}}=3 \frac{\mathrm{E}\left[T(t)^{2}\right]}{\mathrm{E}[T(t)]^{2}}>3,
$$

if $T$ is nondeterministic.

(iii) By conditioning on $\mathcal{F}_{\infty}^{T}$ we can easily see that $X$ inherits this property from the Brownian motion.

(iv) We already know that $\mathrm{E}[X(t)]=0$ and that $X$ has uncorrelated increments. Hence, $X$ has weakly stationary increments on $[a, b]$ if and only if $\operatorname{var}(X(t+h)-X(t))$ does not depend on $t$ for all $t, t+h \in[a, b]$. However, by (i),

$$
\begin{aligned}
\operatorname{var}(X(t+h)-X(t)) & =\mathrm{E}[T(t+h)]-\mathrm{E}[T(t)] \\
& =\mathrm{E}[L(1)]\left(\int_{0}^{t+h} k(t+h, s) \mathrm{d} s-\int_{0}^{t} k(t, s) \mathrm{d} s\right) .
\end{aligned}
$$

Part (v) follows from the continuity of $W$ and $T$.

(vi) By the continuity of $X$, both brackets coincide. The last identity is implied by $\langle X\rangle_{t}=$ $\langle W\rangle_{T(t)}=T(t)$.

We now revisit the examples from the previous section. They share the following properties, which are also illustrated by Figures 3 and 4 .

- Continuous trajectories. It is often argued that large changes in stock prices are best explained by jumps. However, some empirical tests based on the singularity spectrum, as in [15] and [17], indicate that there may be no jumps in the stock prices. However, they also show that stock prices have a rougher behavior than typical stochastic volatility 

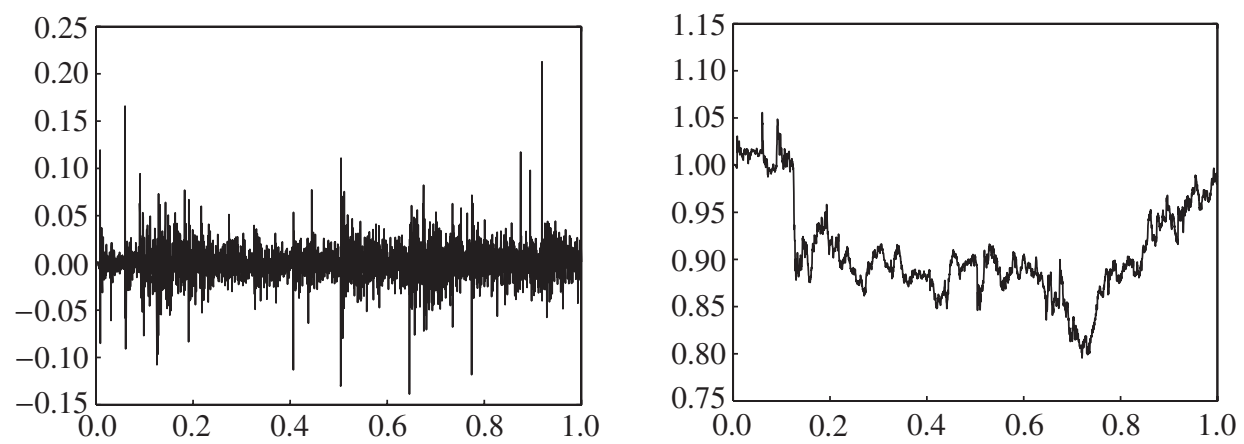

FiguRE 3: Stock (right) and noise, i.e. increments of $X$ (left), based on the Holmgren-Liouville kernel with $H=\frac{3}{4}$ and a gamma process $L$ with $\kappa=\frac{1}{20}$.
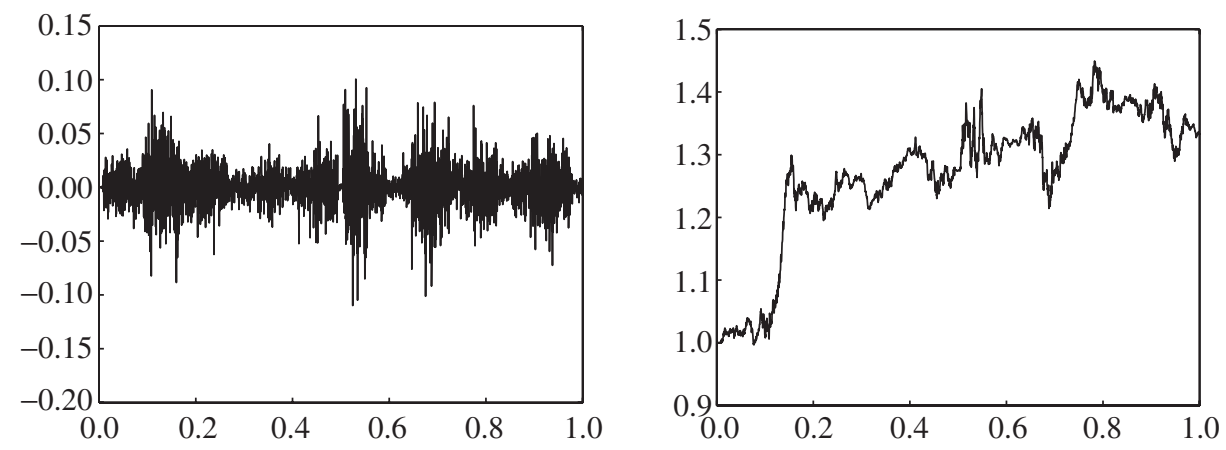

FIGURE 4: Stock (right) and noise, i.e. increments of $X$ (left), based on the short-memory kernel of Example 3 with $\varepsilon=\frac{1}{20}$ and a gamma process $L$ with $\kappa=\frac{1}{20}$.

models whose singularity spectrum reduces to a single point; see also [9, Chapter 7.6]. In this respect, the Holmgren-Liouville fractional kernel looks particularly promising, as the corresponding stock model exhibits a Hölder continuity of order $\left(H-\frac{1}{2}\right) / 2$ at points, where large jumps occur in the subordinator. So, in terms of the roughness of the path, this model is somewhere in between jump models and stochastic volatility models.

- Fat tails. In stock models based on Lévy subordinators, such as the variance gamma model, heavy tails stem from the large jumps of the subordinator. Applying the specific kernels introduced in the examples of the previous section, most mass of a jump of $L$ is eaten up by $T$ shortly after the jump (at least if $H$ is close to $\frac{1}{2}$ or $\varepsilon$ is close to 0 ). Therefore, the tail behavior of the convoluted subordinator is 'similar' to that of the original Lévy subordinator in such situations.

- Volatility clustering. As explained in the previous section, the three example kernels introduce memory into the business time in the sense that a large increase of the business time (and, hence, of the realized volatility) is typically followed by another large one. This effect increases with $H$ or with $\varepsilon$. These two parameters therefore have a simple interpretation, as they admit to adjust between the fatness of the tails and the memory of the integrated volatility. 
We also note that the Holmgren-Liouville fractional subordinator yields weakly stationary increments of the corresponding cumulated noise source $X$, and so does the simple shortmemory kernel of Example 3 after a burn-in-phase until time $t=\varepsilon$.

Remark 1. By the previous proposition we know that the business time $T(t)$ coincides with the realized volatility. While it is obvious, by construction, that $T(t)$ is increasing in $t$, we might ask whether $T(t) / t$ still has some monotonicity properties. We shall show that, for the three example kernels, we can neither expect $T(t) / t$ to be increasing nor decreasing in the convex order, i.e.

$$
t \mapsto \mathrm{E}\left[g\left(\frac{T(t)}{t}\right)\right]
$$

is neither increasing nor decreasing for every convex function $g$ under appropriate assumptions.

(i) Fractional kernel. We calculate

$$
\mathrm{E}\left[\left(\frac{T^{(H)}(t)}{t}\right)^{2}\right]=t^{-2}\left(\operatorname{var}\left(T^{(H)}(t)\right)+\mathrm{E}\left[T^{(H)}(t)\right]^{2}\right)=\mathrm{E}\left[L(1)^{2}\right] t^{2 H-2}+C_{H}^{2} t^{4 H-2}
$$

for some constant $C_{H}$; see Example 1. For $\frac{1}{2}<H<1$, this function is decreasing for small $t$ and increasing for large $t$.

(ii) Short-memory kernel. The same reasoning as for the fractional kernel yields, thanks to Example 3,

$$
\mathrm{E}\left[\left(\frac{T_{\varepsilon}(t)}{t}\right)^{2}\right]= \begin{cases}1+t^{-1}\left(\frac{\mathrm{E}\left[L(1)^{2}\right]}{\mathrm{E}[L(1)]^{2}}-\varepsilon\right)+t^{-2}\left(\frac{\varepsilon^{2}}{4}-\frac{2 \varepsilon \mathrm{E}\left[L(1)^{2}\right]}{3 \mathrm{E}[L(1)]^{2}}\right), & t \geq \varepsilon \\ \frac{\mathrm{E}\left[L(1)^{2}\right]}{\mathrm{E}[L(1)]^{2}} \frac{t}{3 \varepsilon^{2}}+\frac{t^{2}}{4 \varepsilon^{2}}, & t<\varepsilon\end{cases}
$$

which is increasing for small $t$ and decreasing for large $t$, provided $\varepsilon<1$.

(iii) Holmgren-Liouville kernel. For the Holmgren-Liouville kernel (Example 2), we obtain

$$
\mathrm{E}\left[\left(\frac{\tilde{T}^{(H)}(t)}{t}\right)^{2}\right]=1+\frac{\mathrm{E}\left[L(1)^{2}\right]}{\mathrm{E}[L(1)]^{2}} \frac{(H+1)^{2}}{2 H} t^{-1},
$$

which is decreasing in $t$. If $L(t)=\gamma t+J(t)$, where $J$ is an increasing compound Poisson process, we consider the function $g_{\mu}(x)=\max (x, \mu)$ for some $\mu>\gamma / \mathrm{E}[L(1)]$. Then, it is easy to see that

$$
\mathrm{E}\left[g_{\mu}(T(1))\right]>\frac{\gamma}{\mathrm{E}[L(1)]}, \quad \lim _{t \downarrow 0} \mathrm{E}\left[g_{\mu}\left(\frac{T(t)}{t}\right)\right]=\frac{\gamma}{\mathrm{E}[L(1)]},
$$

which shows that $t \mapsto \mathrm{E}\left[g_{\mu}(T(t) / t)\right]$ is not decreasing.

We close this section by providing the characteristic function of the logarithmic stock price. 
Theorem 2. For every $t \geq 0$, the distribution of $\log (S(t))$ is infinitely divisible with characteristic function

$\mathrm{E}[\exp \{\mathrm{i} u \log (S(t))\}]$

$$
\begin{aligned}
=\exp \left\{\mathrm{i} u(\log (S(0))+r t)+\left(\mathrm{i} u\left(\gamma_{0} \mu-\frac{\gamma_{0} \sigma^{2}}{2}\right)-\frac{u^{2} \sigma^{2}}{2}\right) \int_{0}^{t} k(t, s) \mathrm{d} s\right. \\
\left.+\int_{0}^{t} \int_{0}^{\infty}\left(\exp \left\{\left(\mathrm{i} u\left(\mu-\frac{\sigma^{2}}{2}\right)-\frac{u^{2} \sigma^{2}}{2}\right) x k(t, s)\right\}-1\right) v(\mathrm{~d} x) \mathrm{d} s\right\} .
\end{aligned}
$$

Proof. It follows from Theorem 1 , by conditioning on $\mathcal{F}_{\infty}^{T}$, that

$\mathrm{E}[\exp \{\mathrm{i} u \log (S(t))\}]$

$$
\begin{aligned}
& =\mathrm{E}\left[\mathrm{E}\left[\exp \left\{\mathrm{i} u\left(\log (S(0))+r t+\left(\mu-\frac{\sigma^{2}}{2}\right) T(t)+\sigma W(T(t))\right)\right\} \mid \mathcal{F}_{\infty}^{T}\right]\right] \\
& =\exp \{\mathrm{i} u(\log (S(0))+r t)\} \mathrm{E}\left[\exp \left\{\mathrm{i} u\left(\mu-\frac{\sigma^{2}}{2}\right) T(t)-\left(\frac{u^{2} \sigma^{2}}{2}\right) T(t)\right\}\right] \\
& =\exp \{\mathrm{i} u(\log (S(0))+r t)\} \exp \left\{\gamma_{0}\left(\mathrm{i} u\left(\mu-\frac{\sigma^{2}}{2}\right)-\frac{u^{2} \sigma^{2}}{2}\right) \int_{0}^{t} k(t, s) \mathrm{d} s\right. \\
& \left.+\int_{0}^{t} \int_{0}^{\infty}\left(\exp \left\{\left(\mathrm{i} u\left(\mu-\frac{\sigma^{2}}{2}\right)-\frac{u^{2} \sigma^{2}}{2}\right) x k(t, s)\right\}-1\right) v(\mathrm{~d} x) \mathrm{d} s\right\} .
\end{aligned}
$$

This also proves that $\log (S(t))$ is infinitely divisible.

\section{Absence of arbitrage and incompleteness}

We shall now prove that the model, which we introduced in the previous section, is free of arbitrage and incomplete. Recall that the model consists of a riskless bond $B(t)=\mathrm{e}^{r t}$ and stock given by

$$
S(t)=S(0) \exp \left\{r t+\left(\mu-\frac{\sigma^{2}}{2}\right) T(t)+\sigma W(T(t))\right\} .
$$

Here $W$ is a Brownian motion independent of a Lévy subordinator $L$ with Lévy measure $v$ on a filtered probability space $\left(\Omega, \mathcal{F}, \mathcal{F}_{t}, \mathrm{P}\right)_{t \geq 0}$ and

$$
T(t)=\int_{0}^{t} k(t, s) \mathrm{d} L(s) .
$$

As a standing assumption, in this section we shall assume that $v((0, \infty)) \neq 0$. We also fix the investment horizon $\tau \in(0, \infty)$. In order to construct equivalent martingale measures for the market, we first consider, for any continuous function $\eta:[a, b] \rightarrow \mathbb{R}$ such that $[a, b] \subset(0, \infty)$ satisfies $v([a, b]) \neq 0$, the stochastic exponential

$$
Z^{\eta}(t)=\exp \left\{-t \int_{a}^{b}\left(\mathrm{e}^{\eta(x)}-1\right) v(\mathrm{~d} x)+\int_{0}^{t} \int_{a}^{b} \eta(x) \tilde{N}(\mathrm{~d} x, \mathrm{~d} s)\right\} .
$$

Here, $\tilde{N}$ is the compensated jump measure corresponding to $v$. We define a new probability measure $\mathrm{R}^{\eta}$ via the relation

$$
\frac{\mathrm{dR}^{\eta}}{\mathrm{dP}}=Z^{\eta}(\tau)
$$


Under $\mathrm{R}^{\eta}$, the process $L$ is still a Lévy subordinator determined by the pair

$$
v^{\eta}(\mathrm{d} x)=\exp \left\{\mathbf{1}_{[a, b]}(x) \eta(x)\right\} v(\mathrm{~d} x), \quad \gamma_{0}^{\eta}=\gamma_{0} ;
$$

see, e.g. [9, Proposition 9.8].

We next introduce a filtration via

$$
\begin{gathered}
U(t)=T^{-1}(t)=\inf \{s \geq 0 ; T(s) \geq t\}, \\
g_{t}=\sigma(W(s), 0 \leq s \leq t, U(s), 0 \leq s \leq t \wedge \tau) .
\end{gathered}
$$

Note that $W$ is still a Brownian motion with respect to $\left(g_{t}\right)_{t \geq 0}$ under the measure $\mathrm{R}^{\eta}$ and that, for any $0 \leq t \leq \tau, T(t)$ is a stopping time with respect to $\left(g_{t}\right)_{t \geq 0}$, since

$$
\{T(t) \leq s\}=\{U(s) \geq t\} \in g_{s} .
$$

We now define

$$
Z(t)=\exp \left\{-\frac{\mu}{\sigma} W(t \wedge T(\tau))-\frac{\mu^{2}}{2 \sigma^{2}}(t \wedge T(\tau))\right\}
$$

and $Z(\infty):=\lim _{t \rightarrow \infty} Z(t)$. By conditioning on $\mathcal{F}_{\tau}^{T}$ we can easily see that $\mathrm{E}[Z(\infty)]=1$. Therefore, $Z$ is a uniformly integrable martingale with last element $Z(\infty)$ with respect to the filtration $\left(g_{t}\right)_{t \geq 0}$ under the measure $\mathrm{R}^{\eta}$.

Thus, $\mathrm{Q}^{\eta}$ defined by

$$
\frac{\mathrm{dQ}^{\eta}}{\mathrm{dR}^{\eta}}=Z(\infty)
$$

is a probability measure on $(\Omega, \mathcal{F})$ equivalent to P. Thanks to Girsanov's theorem

$$
\tilde{W}(t)=W(t)+\frac{\mu}{\sigma}(t \wedge T(\tau)), \quad t \geq 0
$$

is a Brownian motion with respect to $\mathrm{Q}^{\eta}$. Moreover, the dynamics of $L$ remain unchanged by the second change of measure.

Under $\mathrm{Q}^{\eta}$, the dynamics of the discounted stock are therefore given by

$$
\mathrm{e}^{-r t} S(t)=S(0) \exp \left\{\sigma \tilde{W}(T(t))-\frac{1}{2} \sigma^{2} T(t)\right\},
$$

where $\tilde{W}$ is a Brownian motion and $T(t)$ is a convoluted subordinator under $\mathrm{Q}^{\eta}$ driven by a Lévy subordinator $L$, and the distribution of $L$ is determined by the pair $\left(\gamma_{0}^{\eta}, v^{\eta}\right)$.

It now follows from the optional sampling theorem that $\left\{\mathrm{e}^{-r t} S(t)\right\}_{0 \leq t \leq \tau}$ is a local martingale under $\mathrm{Q}^{\eta}$ with respect to the filtration $\left(g_{T(t)}\right)_{0 \leq t \leq \tau}$. (We can, in fact, easily show that it is a martingale.) Note next that $\mathcal{F}_{t}^{S}=g_{T(t)}$, where $\left(\mathcal{F}_{t}^{S}\right)_{0 \leq t \leq \tau}$ is the filtration generated by the stock $S$. Therefore, under each measure $\mathrm{Q}^{\eta}$, the discounted stock is a (local) martingale with respect to $\left(\mathcal{F}_{t}^{S}\right)$.

Theorem 3. Suppose that $v((0, \infty)) \neq 0$. Then the market $(B(t), S(t))$ is free of arbitrage and incomplete on every finite time horizon $[0, \tau]$ with respect to the information structure given by $\left(\mathcal{F}_{t}^{S}\right)_{0 \leq t \leq \tau}$.

Proof. We have already shown that each of the measures $\mathrm{Q}^{\eta}$ is an equivalent martingale measure for this market. Therefore, the model is free of arbitrage. Incompleteness will follow, if we establish that

$$
\mathrm{Q}_{\mid \mathcal{F}_{\tau} S}^{\eta_{1}} \neq \mathrm{Q}_{\mid \mathcal{F}_{\tau} S}^{\eta_{2}}
$$

for some $\eta_{1}, \eta_{2}$. We take $\eta_{1}=0$ and $\eta_{2}=\mathbf{1}_{[a, b]}$ for some $0<a<b$ such that $v([a, b]) \neq 0$. 
Then,

$$
\begin{aligned}
\mathrm{E}^{\mathrm{Q}^{\eta_{1}}[T(\tau)]} & =\left(\int_{0}^{\tau} k(\tau, s) \mathrm{d} s\right) \mathrm{E}^{\mathrm{Q}^{\eta_{1}}}[L(1)] \\
& =\left(\int_{0}^{\tau} k(\tau, s) \mathrm{d} s\right)\left(\gamma_{0}+\int_{x>0} x v(\mathrm{~d} x)\right) \\
& <\left(\int_{0}^{\tau} k(\tau, s) \mathrm{d} s\right)\left(\gamma_{0}+\int_{x>0} x \exp \left\{\mathbf{1}_{[a, b]}(x)\right\} \nu(\mathrm{d} x)\right) \\
& =\left(\int_{0}^{\tau} k(\tau, s) \mathrm{d} s\right)\left(\gamma_{0}^{\eta_{2}}+\int_{x>0} x v^{\eta_{2}}(\mathrm{~d} x)\right) \\
& =\left(\int_{0}^{\tau} k(\tau, s) \mathrm{d} s\right) \mathrm{E}^{\mathrm{Q}^{\eta_{2}}}[L(1)] \\
& =\mathrm{E}^{\mathrm{Q}^{\eta_{2}}[T(\tau)] .}
\end{aligned}
$$

As $T(\tau)=\langle W\rangle_{T(\tau)}$ is $\mathcal{G}_{T(\tau)}$-measurable and, thus, $\mathcal{F}_{\tau}^{S}$-measurable, the proof is complete.

\section{Option pricing}

In this section we will be concerned with the pricing of European call options in the new model class. We shall assume that we work under risk-neutral dynamics. Hence,

$$
S(t)=S(0) \exp \left\{r t+\sigma W(T(t))-\frac{1}{2} \sigma^{2} T(t)\right\},
$$

where $W$ is a Brownian motion, $T$ is a convoluted subordinator, $\sigma>0$ is a constant, and $r \geq 0$ is the riskless interest rate.

As a first result, by conditioning on $\mathcal{F}_{\tau}^{T}$, we can easily derive a weighted Black-Scholes formula.

Theorem 4. (Weighted Black-Scholes formula.) Let $X=\left(S_{\tau}-K\right)_{+}$be a European call option with strike $K \in \mathbb{R}_{+}$and maturity $\tau$. Then, for the initial fair price $C_{0}(K)$ of $X$, we have

$$
C_{0}(K)=\int_{0}^{\infty}\left[S_{0} \Phi\left(\frac{\log \left(S_{0} / K\right)+\sigma^{2} t / 2}{\sigma \sqrt{t}}\right)-K \mathrm{e}^{-r \tau} \Phi\left(\frac{\log \left(S_{0} / K\right)-\sigma^{2} t / 2}{\sigma \sqrt{t}}\right)\right] F_{T(\tau)}(\mathrm{d} t),
$$

where $\Phi$ is the distribution function of the standard normal distribution and $F_{T(\tau)}$ is the distribution function of $T(\tau)$.

Remark 2. At first glance, the above weighted Black-Scholes formula looks rather useless, since one cannot expect to know the distribution function of $T(\tau)$ in closed form. Indeed, this distribution function depends on the whole path of the driving Lévy subordinator $L$. However, there are effective series expansions for Lévy subordinators (see [4] for the gamma subordinator and [19] for the general case), which can be applied to simulate $L$ (by simulating the jump times $\tau_{j}$ and jump heights $Y_{j}$, neglecting very small jumps). This gives rise to a straightforward simulation of $T$ by setting

$$
\hat{T}(t)=\sum_{j} k\left(t, \tau_{j}\right) Y_{j}
$$

Therefore, option prices can be calculated from this formula by a Monte Carlo simulation of $T$. 
Remark 3. We can also run other models in the business time given by a convoluted subordinator. For instance, we can replace the Brownian motion by a CGMY process. CGYM processes constitute a flexible and popular class of Lévy processes with log-characteristic function

$$
\phi_{\mathrm{CGMY}}(u)=C \Gamma(-Y)\left[(M-\mathrm{i} u)^{Y}-M^{Y}+(G+\mathrm{i} u)^{Y}-G^{Y}\right]
$$

see [8]. Straightforward modifications of the arguments in Section 4 show that the subordinated CGMY model inherits absence of arbitrage and incompleteness from the original CGMY model. Then, prices of European options in the subordinated model are weighted prices of the original CGMY model, analogously to the weighted Black-Scholes formula. Moreover, the characteristic function of the CGMY process subordinated by a convoluted subordinator can be obtained in the same way as for the subordinated Brownian motion in Theorem 2. We just replace the log-characteristic function of the Brownian motion by the one of the CGMY process. This gives rise to option pricing by fast Fourier transform techniques, as discussed below for the Brownian motion case. We note, however, that the business time cannot any longer be interpreted as realized volatility in the CGMY context, and that the corresponding subordinated CGMY model will exhibit jumps.

If the characteristic function of a logarithmic discounted stock price is known analytically then an efficient and simultaneous computation of the price of call options with different strikes is possible by means of Fourier transform methods; see, e.g. [7] and [13]. For a given strike $K$, we denote the $\log$-strike by $k=\log (K)$. Then, for instance, the regularized function

$$
z_{\tau}(k)=\mathrm{e}^{-r \tau} \mathrm{E}\left[\left(\exp \left\{r \tau+\log \left(S_{\tau}\right)\right\}-\mathrm{e}^{k}\right)_{+}\right]-C_{B S}^{\tilde{\sigma}}(k),
$$

where $C_{B S}^{\tilde{\sigma}}(k)$ is the Black-Scholes price of a call option with $\log$-strike $k$, maturity $\tau$, and volatility $\tilde{\sigma}$, can be rewritten as follows (see [9, p. 363]):

$$
z_{\tau}(k)=\frac{1}{2 \pi} \int_{-\infty}^{+\infty} \mathrm{e}^{-\mathrm{i} v k} \zeta_{\tau}(v) \mathrm{d} v,
$$

where

$$
\zeta_{\tau}(v)=\mathrm{e}^{\mathrm{i} v r \tau} \frac{\Phi_{\tau}(v-\mathrm{i})-\Phi_{\tau}^{\tilde{\sigma}}(v-\mathrm{i})}{\mathrm{i} v(1+\mathrm{i} v)}
$$

Here $\Phi_{\tau}$ denotes the characteristic function of $\log \left(S_{\tau} / B_{\tau}\right)$ and $\Phi_{\tau}^{\tilde{\sigma}}$ is the characteristic function of a normal random variable with mean $-\tilde{\sigma}^{2} \tau / 2$ and variance $\tilde{\sigma}^{2} \tau$.

Application of Theorem 2 with $\mu=r=0$ yields

$$
\begin{aligned}
\Phi_{t}(u)=\exp \{\mathrm{i} u \log S(0)\} \exp \{ & -\gamma_{0} \frac{\sigma^{2} u}{2}(\mathrm{i}+u) \int_{0}^{t} k(t, s) \mathrm{d} s \\
& \left.+\int_{0}^{t} \int_{0}^{\infty}\left(\exp \left\{-\frac{u \sigma^{2}}{2}(\mathrm{i}+u) x k(t, s)\right\}-1\right) v(\mathrm{~d} x) \mathrm{d} s\right\} .
\end{aligned}
$$

Of course, an efficient application of the fast Fourier transform technique requires that the double integral in the expression of $\Phi_{t}$ can be computed. We next give some examples where this is possible. 
Example 5. In the case of a stable subordinator with $\gamma_{0}=0$ and $v(\mathrm{~d} x)=\mathbf{1}_{\{x>0\}} c x^{-\alpha-1} \mathrm{~d} x$ for some $c>0$ and $0<\alpha<1$, we can easily see that the double integral in (3) simplifies to

$$
\begin{aligned}
& \int_{0}^{t} \int_{0}^{\infty}\left(\exp \left\{-\frac{u \sigma^{2}}{2}(\mathrm{i}+u) x k(t, s)\right\}-1\right) v(\mathrm{~d} x) \mathrm{d} s \\
& \quad=\int_{0}^{t}|k(t, s)|^{\alpha} \mathrm{d} s \int_{0}^{\infty}\left(\exp \left\{-\frac{u \sigma^{2}}{2}(\mathrm{i}+u) x\right\}-1\right) v(\mathrm{~d} x) .
\end{aligned}
$$

The second integral can be computed in closed form; see, e.g. [9, p. 94]. The first integral is easily performed for the kernels from Examples 2-3. For example, with a slight modification of Example 2, namely with the kernel

$$
k(t, s)=\left(\alpha\left(H-\frac{1}{2}\right)+1\right)\left(1-\frac{s}{t}\right)_{+}^{H-1 / 2},
$$

we obtain

$$
\int_{0}^{t}|k(t, s)|^{\alpha} \mathrm{d} s=t .
$$

Therefore, the corresponding process has the same marginal distribution as an $\alpha$-stable subordinator, but a more involved dependency structure. In a similar way, (3) can be evaluated efficiently for many kernels (at least numerically), if a stable subordinator is applied. However, the corresponding stock model does not have a second moment, which appears to be a drawback in financial modeling.

The next theorem yields $\Phi_{t}$ in the case of a tempered stable subordinator. Here, we require an explicit choice of the kernel to facilitate the computations.

Theorem 5. Suppose that $L$ is a gamma subordinator $(\alpha=0)$ or a tempered stable subordinator $(0<\alpha<1)$, as defined in (1)-(2), and that $k(t, s)=k_{\varepsilon}(t, s)$ is the short-memory kernel of Example 3. Then,

$$
\begin{aligned}
\log \left(\Phi_{t}(u)\right)= & \left((t-\varepsilon)_{+}+\frac{\min (t, \varepsilon)}{1+\alpha}+\frac{2 \varepsilon(1-\alpha)}{\kappa u \sigma^{2}(\mathrm{i}+u)(1+\alpha)}\right) \Psi_{\alpha, \kappa, \hat{\sigma}^{2}(t),-\hat{\sigma}^{2}(t) / 2}(u) \\
& +\mathrm{i} u \log (S(0))+\min (t, \varepsilon) \frac{1-\alpha}{\kappa(1+\alpha)},
\end{aligned}
$$

where $\hat{\sigma}^{2}(t)=\sigma^{2} \min (t, \varepsilon) / \varepsilon$ and

$$
\Psi_{\alpha, \kappa, \sigma^{2}, \theta}(u)= \begin{cases}-\frac{1}{\kappa} \log \left(1+\frac{u^{2} \sigma^{2} \kappa}{2}-\mathrm{i} \theta \kappa u\right), & \alpha=0, \\ \frac{1-\alpha}{\kappa \alpha}\left(1-\left(1+\frac{\kappa\left(u^{2} \sigma^{2} / 2-\mathrm{i} \theta u\right)}{1-\alpha}\right)^{\alpha}\right), & 0<\alpha<1,\end{cases}
$$

is the characteristic exponent of the variance gamma process or the normal tempered stable process with parameters $\alpha, \kappa, \sigma$, and $\theta$.

Proof. We calculate the double integral

$$
\int_{0}^{t} \int_{0}^{\infty}\left(\exp \left\{-\frac{u \sigma^{2}}{2}(\mathrm{i}+u) x k_{\varepsilon}(t, s)\right\}-1\right) v(\mathrm{~d} x) \mathrm{d} s,
$$


with the Lévy measures introduced in (1)-(2), in several steps. Firstly, we may change the order of integration by an application of Fubini's theorem and obtain, by direct integration, for $t<\varepsilon$,

$$
\begin{aligned}
\int_{0}^{t}\left(\exp \left\{-\frac{u \sigma^{2}}{2}(\mathrm{i}+u) x k_{\varepsilon}(t, s)\right\}-1\right) \mathrm{d} s \\
\quad=\int_{0}^{t}\left(\exp \left\{-\frac{u \sigma^{2}}{2}(\mathrm{i}+u) x \frac{t-s}{\varepsilon}\right\}-1\right) \mathrm{d} s \\
=\frac{2 \varepsilon}{u \sigma^{2}(\mathrm{i}+u) x}\left(1-\exp \left\{-\frac{u \sigma^{2}}{2}(\mathrm{i}+u) x \frac{t}{\varepsilon}\right\}\right)-t
\end{aligned}
$$

and, for $t \geq \varepsilon$,

$$
\begin{aligned}
\int_{0}^{t}\left(\exp \left\{-\frac{u \sigma^{2}}{2}(\mathrm{i}+u) x k_{\varepsilon}(t, s)\right\}-1\right) \mathrm{d} s \\
=(t-\varepsilon)\left(\exp \left\{-\frac{u \sigma^{2}}{2}(\mathrm{i}+u) x\right\}-1\right)+\int_{t-\varepsilon}^{t}\left(\exp \left\{-\frac{u \sigma^{2}}{2}(\mathrm{i}+u) x \frac{t-s}{\varepsilon}\right\}-1\right) \mathrm{d} s \\
=(t-\varepsilon)\left(\exp \left\{-\frac{u \sigma^{2}}{2}(\mathrm{i}+u) x\right\}-1\right) \\
\quad+\frac{2 \varepsilon}{u \sigma^{2}(\mathrm{i}+u) x}\left(1-\exp \left\{-\frac{u \sigma^{2}}{2}(\mathrm{i}+u) x\right\}\right)-\varepsilon .
\end{aligned}
$$

Hence,

$$
\begin{aligned}
\int_{0}^{t}\left(\exp \left\{-\frac{u \sigma^{2}}{2}(\mathrm{i}+u) x k_{\varepsilon}(t, s)\right\}-1\right) \mathrm{d} s \\
=(t-\varepsilon)_{+}\left(\exp \left\{-\frac{u \sigma^{2}}{2}(\mathrm{i}+u) x \frac{\min (t, \varepsilon)}{\varepsilon}\right\}-1\right) \\
\quad+\frac{2 \varepsilon}{u \sigma^{2}(\mathrm{i}+u) x}\left(1-\exp \left\{-\frac{u \sigma^{2}}{2}(\mathrm{i}+u) x \frac{\min (t, \varepsilon)}{\varepsilon}\right\}-\frac{u \sigma^{2}(\mathrm{i}+u) x}{2} \frac{\min (t, \varepsilon)}{\varepsilon}\right) .
\end{aligned}
$$

Now recall that the relevant Lévy measure $v$ is defined, for $0 \leq \alpha<1$ and $\kappa>0$, by

$$
v(A)=v_{\kappa, \alpha}(A)=\int_{A} \frac{1}{\Gamma(1-\alpha)}\left(\frac{1-\alpha}{\kappa}\right)^{1-\alpha} \frac{\mathrm{e}^{-(1-\alpha) x / \kappa}}{x^{1+\alpha}} \mathrm{d} x .
$$

We calculate, by integration by parts,

$$
\begin{gathered}
\int_{0}^{\infty} \frac{2 \varepsilon}{u \sigma^{2}(\mathrm{i}+u) x}\left(1-\exp \left\{-\frac{u \sigma^{2}}{2}(\mathrm{i}+u) x \frac{\min (t, \varepsilon)}{\varepsilon}\right\}-\frac{u \sigma^{2}(\mathrm{i}+u) x}{2} \frac{\min (t, \varepsilon)}{\varepsilon}\right) v_{\kappa, \alpha}(\mathrm{d} x) \\
=\int_{0}^{\infty} \frac{2 \varepsilon}{u \sigma^{2}(\mathrm{i}+u) x}\left(1-\exp \left\{-\frac{u \sigma^{2}}{2}(\mathrm{i}+u) x \frac{\min (t, \varepsilon)}{\varepsilon}\right\}-\frac{u \sigma^{2}(\mathrm{i}+u) x}{2} \frac{\min (t, \varepsilon)}{\varepsilon}\right) \\
\quad \times \frac{1}{\Gamma(1-\alpha)}\left(\frac{1-\alpha}{\kappa}\right)^{1-\alpha} \frac{\mathrm{e}^{-(1-\alpha) x / \kappa}}{x^{1+\alpha}} \mathrm{d} x
\end{gathered}
$$




$$
\begin{aligned}
= & \frac{2 \varepsilon((1-\alpha) / \kappa)^{1-\alpha}}{u \sigma^{2}(\mathrm{i}+u) \Gamma(1-\alpha)} \int_{0}^{\infty}\left(\frac{-1}{1+\alpha} \frac{\mathrm{d}}{\mathrm{d} x} x^{-1-\alpha}\right) \mathrm{e}^{-(1-\alpha) x / \kappa} \\
& \times\left(1-\exp \left\{-\frac{u \sigma^{2}}{2}(i+u) x \frac{\min (t, \varepsilon)}{\varepsilon}\right\}\right. \\
= & \left.\frac{2 \varepsilon((1-\alpha) / \kappa)^{1-\alpha}}{u \sigma^{2}(\mathrm{i}+u) \Gamma(1-\alpha)(1+\alpha)}-\frac{u \sigma^{2}(\mathrm{i}+u) x}{2} \frac{\min (t, \varepsilon)}{\varepsilon}\right) \mathrm{d} x \\
& \times \int_{0}^{\infty} \frac{\mathrm{e}^{-(1-\alpha) x / \kappa}}{x^{1+\alpha}}\left(\frac{1-\alpha}{\kappa} \frac{u \sigma^{2}(\mathrm{i}+u) x}{2} \frac{\min (t, \varepsilon)}{\varepsilon}\right. \\
& +\left(\frac{1-\alpha}{\kappa}+\frac{u \sigma^{2}}{2}(\mathrm{i}+u) \frac{\min (t, \varepsilon)}{\varepsilon}\right) \\
= & \left(\frac{2 \varepsilon(1-\alpha)}{\kappa u \sigma^{2}(\mathrm{i}+u)(1+\alpha)}+\frac{\min (t, \varepsilon)}{1+\alpha}\right) \\
& \times \int_{0}^{\infty}\left(\exp \left\{-\frac{u \sigma^{2}}{2}(\mathrm{i}+u) x \frac{\min (t, \varepsilon)}{\varepsilon}\right\}-1\right) v_{\kappa, \alpha}(\mathrm{d} x) \\
& \left.\left.\left.+\min (t, \varepsilon) \frac{\min (t, \varepsilon)}{\kappa(1+\alpha) \Gamma(1-\alpha)}\right\}-1\right)\right) \mathrm{d} x \\
\kappa & \frac{1-\alpha}{\kappa}\left(\frac{1-\alpha}{\kappa}\right)_{0}^{1-\alpha} \frac{\mathrm{e}^{-(1-\alpha) x / \kappa}}{x^{\alpha}} \mathrm{d} x .
\end{aligned}
$$

The last integral can be substituted to a gamma integral, whence

$$
\min (t, \varepsilon) \frac{1-\alpha}{\kappa(1+\alpha) \Gamma(1-\alpha)}\left(\frac{1-\alpha}{\kappa}\right)^{1-\alpha} \int_{0}^{\infty} \frac{\mathrm{e}^{-(1-\alpha) x / \kappa}}{x^{\alpha}} \mathrm{d} x=\min (t, \varepsilon) \frac{1-\alpha}{\kappa(1+\alpha)} .
$$

Combining these considerations with (4) we obtain

$$
\begin{aligned}
& \int_{0}^{t} \int_{0}^{\infty}\left(\exp \left\{-\frac{u \sigma^{2}}{2}(\mathrm{i}+u) x k_{\varepsilon}(t, s)\right\}-1\right) v(\mathrm{~d} x) \mathrm{d} s \\
&=\min (t, \varepsilon) \frac{1-\alpha}{\kappa(1+\alpha)}+\left((t-\varepsilon)_{+}+\frac{2 \varepsilon(1-\alpha)}{\kappa u \sigma^{2}(\mathrm{i}+u)(1+\alpha)}+\frac{\min (t, \varepsilon)}{1+\alpha}\right) \\
& \times \int_{0}^{\infty}\left(\exp \left\{-\frac{u \sigma^{2}}{2}(\mathrm{i}+u) x \frac{\min (t, \varepsilon)}{\varepsilon}\right\}-1\right) v_{\kappa, \alpha}(\mathrm{d} x) .
\end{aligned}
$$

Taking into account the fact that the characteristic exponent of a variance gamma process $(\alpha=0)$ or a normal tempered stable process $(0<\alpha<1)$ is, by definition,

$$
\Psi_{\alpha, \kappa, \sigma, \theta}(u)=\int_{0}^{\infty}\left(\exp \left\{-\frac{u^{2} \sigma^{2}}{2} x+\mathrm{i} \theta u x\right\}-1\right) v_{\kappa, \alpha}(\mathrm{d} x),
$$

the first assertion is proved. The explicit form of $\Psi_{\alpha, \kappa, \sigma, \theta}$ can, e.g. be found in [9, p. 117].

We also state the log-characteristic function for the gamma case and the tempered stable case, if the Holmgren-Liouville fractional kernel is applied. The representation follows directly from (3). As it requires one additional integration, a numerical evaluation of the fast Fourier transform methods is slower, but still feasible. 

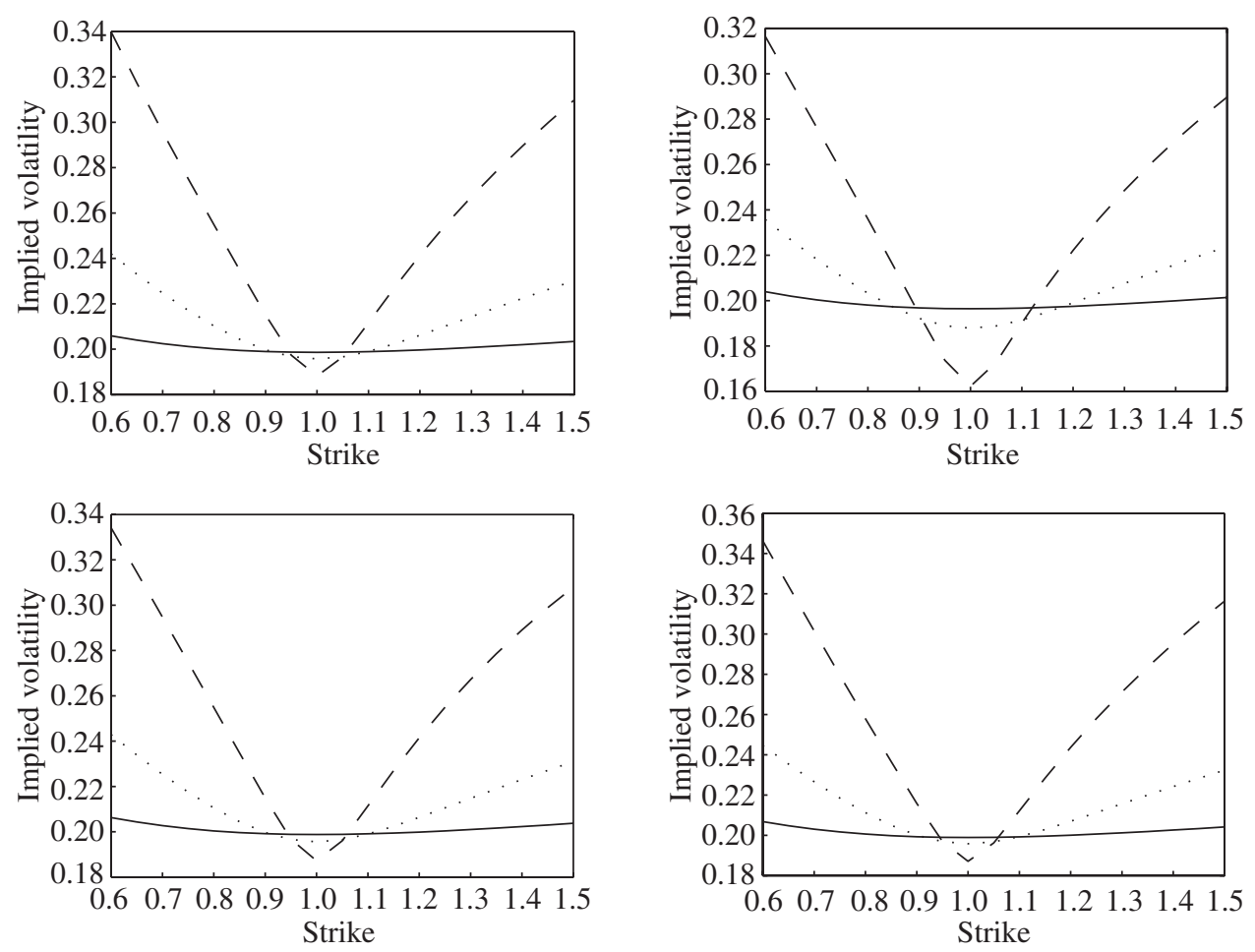

FIGURE 5: Implied volatility smile for 1 (solid line), 0.3 (dotted line), and 0.1 (dashed line) year maturities. Top left: variance gamma model with $r=0, \sigma=0.2, \kappa=0.05$, and $S(0)=1$. Top right: short-memory kernel with $\varepsilon=0.05$ driven by a gamma subordinator with the same parameters. Bottom: damped Holmgren-Liouville kernel with $H=0.7$ (left) and $H=0.9$ (right) driven by a gamma subordinator with the same parameters.

Theorem 6. Suppose that $L$ is a gamma subordinator $(\alpha=0)$ or a tempered stable subordinator $(0<\alpha<1)$, as defined in (1)-(2), and that $k(t, s)=\tilde{z}_{H}(t, s)$ is the Holmgren-Liouville fractional kernel of Example 2. Then,

$$
\log \left(\Phi_{t}(u)\right)=\int_{0}^{t} \Psi_{\alpha, \kappa, \hat{\sigma}^{2}(s, t),-\hat{\sigma}^{2}(s, t) / 2}(u) \mathrm{d} s,
$$

where $\hat{\sigma}^{2}(s, t)=\sigma^{2}\left(H+\frac{1}{2}\right)(1-s / t)^{H-1 / 2}$ and $\Psi_{\alpha, \kappa, \sigma, \theta}(u)$ was defined in the previous theorem.

Finally, we illustrate the implied volatility smile of models based on different kernels and driven by a gamma process. Figure 5 shows that, with the short-memory kernel of Example 3 and the damped Holmgren-Liouville kernel of Example 2, the smile flattens out quickly with increasing maturity as in the variance gamma model. Indeed, the four smiles for the different models resemble each other very closely. From this we may conclude that, in particular for the damped Holmgren-Liouville kernel, the marginal distributions of the model do not vary much in the memory parameter $H$. Therefore, the prices of plain vanilla options do not change significantly for different values of $H$. It is, however, expected that the prices of path-dependent 
options, such as barrier options, will strongly depend on the dependency structure of the model induced by the memory parameter $H$. This topic will be further pursued in future research.

\section{References}

[1] BarndorfF-Nielsen, O. E. And ShePhard, N. (2001). Non-Gaussian Ornstein-Uhlenbeck-based models and some of their uses in financial economics. J. Roy. Statist. Soc. B 63, 167-241.

[2] Baudoin, F. and Nualart, D. (2003). Equivalence of Volterra processes. Stoch. Process. Appl. 107, $327-350$.

[3] Bender, C. And Marquardt, T. (2008). Stochastic calculus for convoluted Lévy processes. Bernoulli 14, 499-518.

[4] Bondesson, L. (1982). On simulation from infinitely divisible distributions. Adv. Appl. Prob. 14, 855-869.

[5] Brockwell, P. J. (2001). Lévy-driven CARMA processes. Ann. Inst. Statist. Math. 52, 113-124.

[6] Brockwell, P. J. ANd Marquardt, T. (2005). Lévy-driven and fractionally integrated ARMA processes with continuous time parameter. Statistica Sinica 15, 477-494.

[7] Carr, P. And Madan, D. (1998). Option valuation using the fast Fourier transform. J. Comput. Finance 2, 61-73.

[8] Carr, P., Geman, H., Madan, D. B. And Yor, M. (2003). Stochastic volatility for Lévy processes. Math. Finance 13, 345-382.

[9] Cont, R. And Tankov, P. (2004). Financial Modelling with Jump Processes. Chapmann and Hall, Boca Raton, FL.

[10] Eberlein, E. (2001). Application of generalized hyperbolic Lévy motions to finance. In Lévy Processes, eds O. Barndorff-Nielsen et al., Birkhäuser, Boston, pp. 319-336.

[11] Gander, M. P. S. And Stephens, D. A. (2007). Simulation and inference for stochastic volatility models driven by Lévy processes. Biometrika 94, 627-646.

[12] Karatzas, I. And Shreve, S. E. (1991). Brownian Motion and Stochastic Calculus (Graduate Texts Math. 113), 2nd edn. Springer, New York.

[13] LEWIS, A. (2001). A simple option formula for general jump-diffusion and other exponential Lévy processes. Tech. Rep. Available at www.optioncity.net/publications.htm.

[14] Madan, D. And Milne, F. (1991). Option pricing with variance gamma martingale components. Math. Finance 1, 39-55.

[15] Mandelbrot, B. B., Calvet, L. and Fisher, A. (1997). The multifractality of the Deutschmark/US Dollar exchange rate. Cowles Foundation of Economics Discussion Papers.

[16] Marquardt, T. (2006). Fractional Lévy processes with an application to long memory moving average processes. Bernoulli 12, 1099-1126.

[17] Muzy, J., Delour, J. ANd Bacry, E. (2000). Modeling fluctuations of financial time series: from cascade processes to stochastic volatility models. Europ. J. Phys. B 17, 537-548.

[18] Norros, I., Valkeila, E. And Virtamo, J. (1999). An elementary approach to a Girsanov formula and other analytical results on fractional Brownian motions. Bernoulli 5, 571-587.

[19] Rosinski, J. (2001). Series representations of Lévy processes from the perspective of point processes. In Lévy Processes, eds O. E. Barndorff-Nielsen et al., Birkhäuser, Boston, pp. 401-415.

[20] Sato, K.-I. (1999). Lévy Processes and Infinitely Divisible Distributions. Cambridge University Press.

[21] Sato, K.-I. (2006). Addtitive processes and stochastic integrals. Illinois J. Math 50, 825-851. 